\title{
Serie inicial de prostatectomía radical laparoscópica en un hospital general: revisión y comparativa de series nacionales e internacionales.
}

\author{
Crego Tapias M, Puig Duran P, Juaneda Castell B, Segura Forcada J, Vallejo Gil C, \\ Cortadellas Angel Ll.
}

Servicio de Urología. Hospital General de Granollers. Barcelona.

Actas Urol Esp. 2008;32(9):888-893

\begin{abstract}
RESUMEN
SERIE INICIAL DE PROSTATECTOMÍA RADICAL LAPAROSCÓPICA EN UN HOSPITAL GENERAL: REVISIÓN Y COMPARATIVA DE SERIES NACIONALES E INTERNACIONALES

Serie inicial de prostatectomía radical laparoscópica en un hospital general: revisión y comparativa de series nacionales e internacionales.

Introducción y objetivos: La prostatectomía radical laparoscópica es una técnica que ha demostrado unos resultados oncológicos y funcionales competitivos, por lo que se ha convertido en un procedimiento rutinario en muchos centros punteros. No obstante, la dificultad de aprendizaje y su coste inicial, pueden cuestionar su implantación como técnica rutinaria, en centros que no sean de referencia.

Realizamos un estudio descriptivo de nuestra serie inicial analizando la técnica quirúrgica, resultados posquirúrgicos inmediatos, y oncológicos y funcionales a corto plazo, comparándolos con otras series en centros nacionales e internacionales.

Métodos: De febrero 2006 a agosto 2007 realizamos 69 procedimientos laparoscópicos en nuestro centro, de los cuales 34 fueron prostatectomías radicales. La edad media de la serie fue de 63 años (50-72), con un peso prostático medio por ecografía transrectal de 35 gr. (17-72), y una mediana de PSA de $6 \mathrm{ng} / \mathrm{ml}$ (4-35). Los estadios clínicos fueron: T1c 59\%, T2a 12\%, T2b 17\%, T2c 12\%; con Gleason: 2+2 en $3 \%, 3+3$ en $35 \%, 3+4$ en $15 \%, 4+3$ en $44 \%, 4+5$ en $3 \%$.

Resultados: De los 34 casos iniciados por laparoscopia, se terminaron 30, con un tiempo quirúrgico medio de 261 minutos (150-380). Se convirtieron 4 casos a cirugía abierta, 1 por enfisema subcutáneo, 1 por prolongación del tiempo quirúrgico, 1 por sangrado, 1 por lesión rectal.

Los estadios patológicos fueron: pT2a 3\%, pT2b 18\%, pT2c 41\%, pT3a 32\%, pT3b 6\%. Con Gleason de: 3+3 en 26\%, 3+4 en 14\%, 4+3 en 47\%, 4+4 en 9\%, 4+5 en 3\%. Obtuvimos un 29\% de márgenes positivos (50\% pT3a, 20\% pT3b, 20\% pT2b, 10\% pT2c).

La continencia (0-1 compresas) al mes, fue del $62 \%$, llegando al $84 \%$ a los 6 meses. La erección al mes, se conservó en un $50 \%$ de los pacientes, en los cuatro casos de disección intrafascial.

Conclusiones: la prostatectomía radical laparoscópica es una técnica de difícil aprendizaje, y exige un tiempo quirúrgico inicial superior a la cirugía abierta. No obstante, comprobamos que se trata de una técnica segura, con unos resultados oncológicos y funcionales competitivos, una recuperación temprana y un escaso sangrado, incluso en series iniciales. Por ello, también debería considerarse una opción para el tratamiento del cáncer de próstata en centros de segundo nivel.

Palabras clave: Cáncer próstata. Prostatectomía radical. Laparoscopia.
\end{abstract}

\section{ABSTRACT}

INITIAL SERIES OF RADICAL PROSTATECTOMY IN A GENERAL HOSPITAL: REVIEW AND COMPARATIVE OF NATIONAL AND INTERNATIONAL SERIES.

Introduction and objectives: The radical prostatectomy is a technique that has shown competitive oncological and functional results, that is why it has become to be a routine procedure in many leading centers. Nevertheless, the difficulty of learning and its initial cost, could question its implantation as a routine technique in centers that are not a point of reference.

We have carried out a descriptive study of our initial series analyzing the surgical technique, post surgical immediate results, and oncological and functional short-term outcomes, comparing them with other series in national and international centers.

Methods: From February, 2006 to August, 2007 we performed 69 laparoscopical procedures in our center, of which 34 were radical prostatectomies. The average age of the series were 63 years (50-72), with a prostate average weight by transrectal ultrasound of 35 gr. (17-72), and a median PSA of $6 \mathrm{ng} / \mathrm{ml}$ (4-35). The clinical stages were: T1c 59\%, T2a 12\%, T2b 17\%, T2c $12 \%$; with Gleason combined of: $2+2$ in $3 \%, 3+3$ in $35 \%, 3+4$ in $15 \%, 4+3$ in $44 \%, 4+5$ in $3 \%$.

Results: Out of the 34 cases initiated by laparoscopy, 30 were concluded laparoscopically, with a surgical average time of 261 minutes (150380). The pathological stages were: pT2a 3\%, pT2b 18\%, pT2c 41\%, pT3a $32 \%$, pT3b 6\%. With Gleason of: $3+3$ in $26 \%$, $3+4$ in $14 \%$, $4+3$ in $47 \%, 4+4$ in $9 \%, 4+5$ in 3\%. We obtained $29 \%$ of positive margins ( $50 \%$ pT3a, $20 \%$ pT3b, 20\% pT2b, $10 \%$ pT2c). Four cases were converted to open surgery, one due to subcutaneous emphysema, one to enlarged surgical time, one bleeding, and one rectal injury.

The continence (0-1 pads) at the first month was $62 \%$, reaching $84 \%$ at 6 months.

The erection, remained in $50 \%$ of the patients at the first month, in the four cases of intrafascial dissection.

Conclusions: Laparoscopic radical prostatectomy is a technique of difficult learning, and initially requires higher surgical time than open surgery.Nevertheless, we prove that it is a reliable technique with competitive oncological and functional results, earlyrecovery and little bleeding even in the initial series. Therefore, it should be considered an option for the treatment of prostate cancer also in not leading centers.

Keywords: Prostate cancer. Radical prostatectomy. Laparoscopy. 
$\mathrm{D}$ esde que se describió el primer procedimiento laparoscópico urológico $^{1}$, la integración de la laparoscopia en urología ha sido lentamente progresiva por falta de patología frecuente y técnicamente sencilla.

Es por ello, que la estandarización de esta técnica en urología, ha tenido que esperar una curva de aprendizaje larga en los centros de referencia, para posteriormente extenderse a centros de segundo nivel.

Presentamos la serie inicial de un hospital general con 18 meses de experiencia en ésta técnica. Estudiamos los resultados quirúrgicos, funcionales y oncológicos a tres y seis meses, y los comparamos a series iniciales de centros de referencia nacionales e internacionales.

\section{MÉTODOS}

En febrero de 2006, iniciamos el programa de laparoscopia urológica en nuestro centro. Desde entonces, hemos realizado 69 procedimientos en 18 meses, de los cuales 34 prostatectomías radicales.

Analizamos la serie de prostatectomías radicales, con un seguimiento de 6 meses.

Los criterios de inclusión fueron, como en cirugía abierta, enfermedad localizada por estadiaje clínico (Gleason menor o igual a 7, PSA hasta 20 $\mathrm{ng} / \mathrm{ml}$, tacto rectal no sugestivo de enfermedad extracapsular), sin antecedentes patológicos añadidos de riesgo quirúrgico, y edad menor de 70 años. Dichos criterios fueron personalizados para cada paciente.

La media de edad de la serie fue de 63 años (rango: 50-72), con un peso prostático medio por ecografía transrectal de 35 g (17-72), y una mediana de PSA de 6ng/ml (4-35).

Se realizaron una mediana de 1 biopsia transrectal por paciente (1-3) siendo sextantes, u octantes en los casos de biopsia previa. Los estadios clínicos fueron de T1c 59\% (20), T2a 12\% (4), T2b 17\% (6), T2c $12 \%$ (4). Los valores de Gleason combinado (Gl) fueron de $2+2$ en $3 \%$ (1), $3+3$ en $35 \%$ (12), 3+4 en $15 \%$ (5), 4+3 en 44\% (15), 4+5 en 3\% (1).

Para la técnica quirúrgica realizada, colocamos un trócar de 10/12 mm (óptica $0^{\circ}$ ), y 4 trócares de $5 \mathrm{~mm}$., 2 para el cirujano (tijera monopolar, pinza bipolar/ Ligasure ${ }^{\circledR} /$ Ultracision ${ }^{\circledR}$, porta agujas), y 2 para el ayudante (pinza y aspirador), dispuestos de manera a evitar el cruce de brazos entre ambos.
En todos los casos realizamos un inicio intraperitoneal, accediendo posteriormente al espacio de Retzius.

Se realizó conservación del cuello vesical en 30 casos. En los 4 restantes, la presencia de un lóbulo medio prominente dificultó la disección, obligando a realizar puntos en raqueta en 2 casos. Intentamos acceder al plano de las vesículas seminales siempre antes de seccionar la uretra para evitar entrar en el plano del adenoma. Tras disecar vesículas y deferentes accedemos al plano posterior abriendo la fascia de Denonvilliers. Realizamos una disección interfascial siempre que hallamos un buen plano de disección, lateralmente a la próstata, entre la fascia endopélvica y la fascia periprostática. En 7 casos con criterios de bajo riesgo (PSA menor o igual a 10ng/ml, Gleason menor o igual a 6, estadio T1-2a), y con erección previa, intentamos realizar una disección intrafascial y sin coagulación de los pedículos prostáticos, asumiendo un mayor riesgo de sangrado y de márgenes positivos. Se desestimaron 3 casos durante la cirugía por dificultad técnica para realizar dicha disección.

Comprobamos la liberación completa de la próstata antes de la disección del complejo de Santorini y la uretra.

En los 4 primeros casos, la sección del complejo de Santorini se realizó mediante corte frío para evitar lesiones térmicas desapercibidas en uretra. Los siguientes casos se realizaron con Ligasure ${ }^{\circledR}$ o Ultracision ${ }^{\circledR}$, por mayor sangrado intra y postoperatorio en los casos de sección fría. Añadimos un punto hemostático en el complejo de Santorini tras obtener la pieza.

Hemos realizado en todos los casos una anastomosis continua de inicio a las 9 horas con Monocryl® 3/0.

\section{RESULTADOS}

En los 34 casos realizados, el tiempo quirúrgico medio fue de 261 minutos (150-380). Se convirtieron a cirugía abierta 4 casos (Fig. 1). El primer caso se convirtió por enfisema subcutáneo y aumento de la pCO2 en sangre, secundariamente a una fuga de gas desapercibida por un puerto. En el tercer caso, la conversión fue por sangrado del complejo de Santorini, que no se controló con puntos hemostáticos laparoscópicos. En los siguientes casos, la sección del complejo se realizó siempre con Ultracision ${ }^{\circledR}$ 


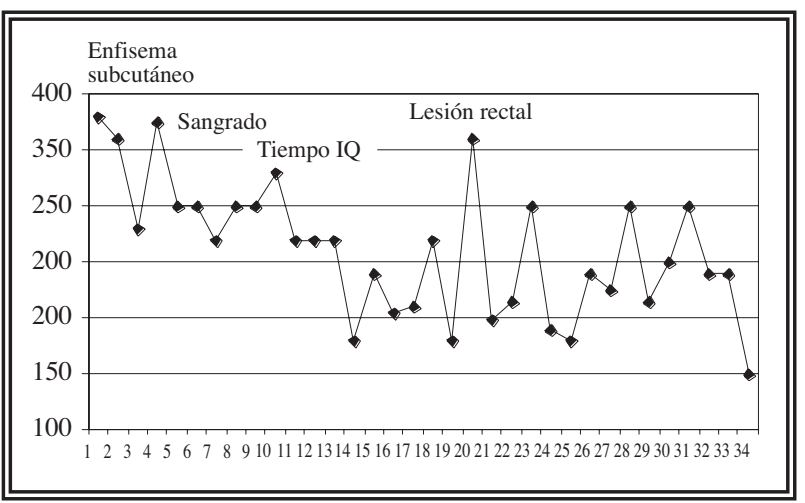

FIGURA 1. Tiempos quirúrgicos y complicaciones en los casos de conversión a cirugía abierta.

o Ligasure®, en lugar de corte frío. En el noveno caso, se convirtió a cirugía abierta por exceso de tiempo quirúrgico en un paciente con un lóbulo medio prominente, que alargó el tiempo de disección de cuello y obligó a realizar varios puntos en raqueta. El caso 21 fue nuestra la última conversión, por una lesión rectal en un paciente con enfermedad extracapsular. Se inició la reparación laparoscópica del recto, pero se continuó abierta por aumento del tiempo quirúrgico, y para mayor seguridad de la sutura.

Ente las complicaciones postquirúrgicas contamos con 1 caso de sangrado que no requirió reintervención; dos casos de fuga urinaria, en dos casos con lóbulo medio prostático, que se solucionaron quitando el globo de la sonda vesical; y una fístula rectal.

Se transfundieron 2 pacientes en total (5.8\%). Los valores de hemoglobina media pre y postquirúrgicos fueron 14,3 g/dl (11-18) y 12,1 g/dl (7-15).

La analgesia necesaria en las primeras horas en reanimación, fue mayoritariamente una combinación de mórficos con AINES (62\%), solamente mórficos (14\%), o solamente AINES (9\%). En un $15 \%$ se realizó anestesia peridural, que en todos los casos se tuvo que combinar con otro analgésico (mórfico 3\%, AINE 12\%).

Los mórficos administrados se mantuvieron una media de 4 horas (0-11), tras las cuales el dolor se controló con AINES (85\%) o catéter peridural (15\%).

Los estadios patológicos de las piezas fueron pT2a en un $3 \%$ (1), pT2b en un $18 \%$ (6), pT2c en un $41 \%$ (14), pT3a en un 32\% (11), pT3b en un 6\% (2). Con unos Gleason de $3+3$ en $26 \%$ (9), $3+4$ en $14 \%$ (5), $4+3$ en $47 \%$ (16), $4+4$ en $9 \%$ (3), $4+5$ en $3 \%$ (1).
Obtuvimos márgenes positivos en un 29\%, de los cuales, un 50\% (5) pT3a, un 20\% (2) pT3b, un 20\% (2) pT2b, y un 10\% (1) pT2c.

Los casos de recidiva bioquímica (PSA $\geq 0,2$ $\mathrm{ng} / \mathrm{ml}$ ) a los 6 meses, son del 15\% (5).

La continencia al mes de la retirada de la sonda vesical, definida como paciente seco, o portador de una compresa diaria es del 62\%, llegando al 84\% a los seis meses (Fig. 2). El resto de pacientes, requieren al mes y los 6 meses respectivamente: 2 compresas $16 \%$ y 10\%, 3 compresas $22 \%$ y $6 \%$. Este último grupo comprende el primer caso realizado, con enfermedad extracapsular y recidiva bioquímica, pendiente de colocación de esfínter artificial después de realizar radioterapia.

En cuanto a la potencia, entre los pacientes seleccionados para preservación de bandeletas, se realizó disección intrafascial unilateral o bilateral en 4 casos. En estos casos, obtuvimos conservación de la erección (considerada como posibilidad para penetración con o sin tratamiento vía oral) en la mitad de los casos.

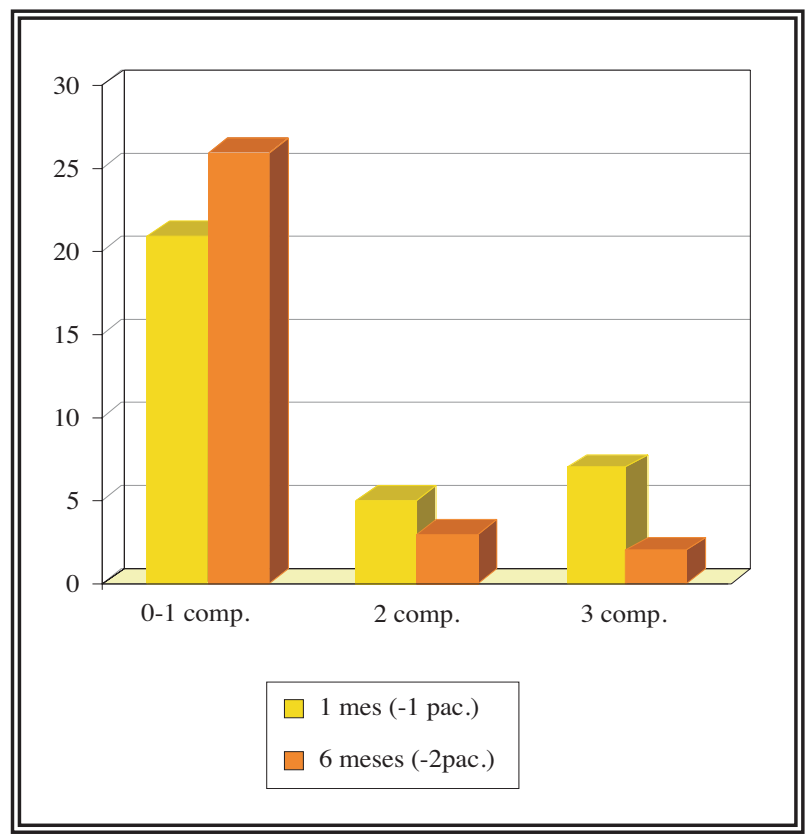

FIGURA 2. Resultados de continencia a 3 y 6 meses.

\section{DISCUSIÓN}

El inicio de la cirugía laparoscópica en centros de segundo nivel, ha sido controvertido ${ }^{2}$, por ser una técnica nueva, de difícil aprendizaje, y con cirugías de alta dificultad técnica, como la prostatectomía radical 
laparoscópica, para la que algunos autores estiman una curva de aprendizaje de un mínimo de unos 60 $\operatorname{casos}^{3,4}$.

Para evaluar los resultados de nuestra serie inicial, los comparamos con las primeras series iniciales publicadas (Tabla 1). Los resultados obtenidos en nuestro centro son equiparables a los obtenidos en centros de referencia, en términos de tiempo quirúrgico, complicaciones y conversión a cirugía abierta $^{5-7}$.

La evaluación de los resultados funcionales, y sobre todo oncológicos, en series iniciales, es difícil dado el escaso tiempo de seguimiento.

En nuestra serie, obtenemos una continencia a los 6 meses de la cirugía, de un 84\%. Los resultados de continencia en las series internacionales publicadas $^{11,14-21}$, con un seguimiento de 6 a 24 meses, es de un 80 a un $95 \%$ (Tabla 2).

La conservación de la erección tras prostatectomía radical laparoscópica no ha sido nuestro objetivo en estos casos iniciales. Pensamos que, al igual que en cirugía abierta, la preservación de bandele- tas exige un dominio de la técnica quirúrgica para evitar un mayor sangrado, el riesgo de márgenes positivos, y un aumento del tiempo quirúrgico.

No obstante, hemos realizado una disección intrafascial, evitando lesionar los haces neurovasculares, en 4 pacientes seleccionados, conservando la erección sin tratamiento o con tratamiento vía oral, en la mitad de los casos.

Los resultados oncológicos son de difícil evaluación en series con poco seguimiento, pero la incidencia de márgenes positivos en pacientes con enfermedad organoconfrinada (3 casos), nos indican que podrían existir recidivas debidas a la técnica quirúrgica.

En series nacionales, la incidencia de márgenes positivos es de un 12 a un $33 \%{ }^{5-7}$. En grandes series internacionales ${ }^{8-10,12,13,18-20}$, se obtienen unos márgenes positivos de un 14 a un $31 \%$ (Tabla 3). En nuestra serie, obtenemos un $29 \%$ de márgenes positivos, de los cuales, un 70\% en pT3a y pT3b.

Hemos considerado como recidiva bioquímica a los 6 meses, los casos con PSA mayor o igual a $0,2 \mathrm{ng} / \mathrm{ml}$.

Tabla 1. Resultados en las series iniciales en Hospital Universitari Germans Trias i Pujol, Hospital Universitario La Paz, Fundació Puigvert, Consorci Sanitari Parc Tauli, Hospital General de Granollers ${ }^{5-7}$.

\begin{tabular}{lcccccccc}
\hline & Casos & Técnica IQ & Tiempo IQ & Conversión & Complicaciones & Márgenes & Continencia 6m & Potencia 6m \\
\hline HUGTP '03 & 27 & Intrap. & 296 & $37 \%$ & $4 \%$ & $33 \%$ & - & $26 \%$ (total) \\
HULP '04 & 147 & Intrap. & 243 & $3 \%$ & $6 \%$ & $33 \%$ & $90 \%(12 \mathrm{~m})$ & $\begin{array}{c}50 \% \text { (preservación } \\
\text { bandeletas) }\end{array}$ \\
FP* '05 & 70 & Intrap. & 240 & - & $4 \%$ & $24 \%$ & $75-96 \%$ & $15 \%$ (total) \\
CSPT ‘06 & 50 & Extrap. & 287 & $22 \%$ & $4 \%$ & $12 \%$ & - & - \\
HGG '07 & 34 & Intrap. & 261 & $11 \%$ & $3 \%$ & $29 \%$ & $84 \%$ & $50 \%$ (preservación \\
bandeletas)
\end{tabular}

(*) LXX Congreso Nacional de Urología. Prostatectomía radical laparoscópica. Análisis de Nuestra exeperiencia inicial. Rosales A, Salvador J, Palou J, Alcaraz A, De Graeve N, Segarra J, Villavicencio H.

Tabla 2. Porcentaje de márgenes en series internacionales ${ }^{8-10,12,13,18-20}$

\begin{tabular}{|c|c|c|c|c|c|c|c|c|}
\hline & $\begin{array}{c}\text { Guillonneau } \\
\text { Montsouris }\end{array}$ & $\begin{array}{c}\text { Rassweiler } \\
\text { Heilbron }\end{array}$ & $\begin{array}{c}\text { Menon } \\
\text { Vattikuti }\end{array}$ & $\begin{array}{c}\text { Rhee } \\
\text { Burlington }\end{array}$ & $\begin{array}{c}\text { Fromont } \\
\text { Montsouris }\end{array}$ & $\begin{array}{l}\text { Abbou } \\
\text { Créteil }\end{array}$ & $\begin{array}{c}\text { Stolzenbourg } \\
\text { Leipzig }\end{array}$ & $\begin{array}{c}\text { Gaston } \\
\text { Bordeaux }\end{array}$ \\
\hline Pacientes & 1000 & 957 & 100 & 139 & 100 & 165 & 700 & 413 \\
\hline márgenes + (\%) & 19,2 & 22,6 & 15 & 13.6 & - & 29,7 & 10,8 & 30,7 \\
\hline pT2a & 6,9 & 3,2 & 9.5 & 4,2 (pT2) & 7,9 (pT2) & 4,8 & 2,2 & 7,4 \\
\hline pT2b & 18,6 & 9 & 11 & - & - & 20,6 & 14,8 & 21 \\
\hline pT2c & - & - & - & - & - & & 13,1 & 24 \\
\hline pT3a & 30 & 34,5 & 40 & 79 (рT3) & 41,7 (pT3) & 42,3 & 24,5 & 43 \\
\hline pT3b & 34 & 48,8 & 44 & & & 50 & 50,6 & 46 \\
\hline
\end{tabular}


Tabla 3. Porcentaje de continencia y potencia en series internacionales ${ }^{11,14-21}$.

\begin{tabular}{lccccc}
\hline Centro/Autor & $\begin{array}{c}\text { Pacientes } \\
\text { totales }\end{array}$ & $\begin{array}{c}\text { Pacientes } \\
\text { evaluados }\end{array}$ & $\begin{array}{c}\text { Continencia(\%)/ } \\
\text { Seguimiento(m) }\end{array}$ & $\begin{array}{c}\text { Conservación/ } \\
\text { Bandeletas }\end{array}$ & $\begin{array}{c}\text { Potencia(\%)/ } \\
\text { Seguimiento(m) }\end{array}$ \\
\hline Salomón/Créteil & 235 & 100 & $90 / 12$ & 17 & $58,8 / 12$ \\
Anastasiadis/Créteil & 230 & 106 & $89 / 12$ & 77 & $53 / 12$ \\
Rassweiler/Heilbron & 850 & 310 & $97,7 / 24$ & 41 & $67 / 12$ \\
Guillonneau/Montsouris & 550 & 255 & $82,3 / 12$ & 47 & $66 / 4$ \\
Rozet/Montsouris & 600 & 498 & $84 / 12$ & 26 & $43 / 6$ \\
Roumeguere/Brussels & 85 & 52 & $80,7 / 12$ & 34 & $65,3 / 12$ \\
Stolzenbourg/Leipzig & 700 & 420 & $92 / 12$ & - & $47,1 / 6$ \\
Menon/Vatikutti & 100 & 100 & 91,6 & 137 & $59 / 6$ \\
Gaston/Bordeaux & 425 & 202 & $95 / 12$ & $58,5 / 12$ \\
\hline
\end{tabular}

De éstos, 3 son pacientes con afectación extracapsular en la pieza y márgenes positivos. Los 2 restantes, son pT2c con márgenes negativos, uno de los cuales mantinene un PSA de $0,3 \mathrm{ng} / \mathrm{ml}$ sin progresión. El otro paciente, con un PSA prequirúrgico de $13 \mathrm{ng} / \mathrm{ml}$, hace sospechar la posibilidad de enfermedad diseminada.

\section{CONCLUSIONES}

La prostatectomía radical es la cirugía abierta más frecuente en nuestra especialidad. La alternativa laparoscópica es una de las cirugías técnicamente más complejas en urología, y requiere una curva de aprendizaje larga ${ }^{3,4}$. Los resultados oncológicos son similares a los que se obtienen en la cirugía abierta ${ }^{22}$. Los resultados funcionales son, como mínimo, equiparables a los de la cirugía abierta a largo plazo ${ }^{24}$. No obstante la laparoscopia ofrece una menor morbilidad postoperatoria, traducida en un menor sangrado quirúrgico y un tiempo de recuperación posquirúrgico menor ${ }^{22,23}$.

En nuestra experiencia, la prostatectomía radical laparoscópica es una técnica que exige un mayor tiempo quirúrgico respecto a la cirugía abierta. Como en el aprendizaje de cualquier técnica quirúrgica, deben asumirse complicaciones y falta de radicalidad, en algunos casos, debido al aprendizaje.

No obstante, observamos un escaso sangrado intraoperatorio, un menor dolor postoperatorio, y una rápida recuperación funcional.

Pensamos que las ventajas que ofrece la prostatectomía radical laparoscópica deben ofrecerse tanto en centros de referencia, como en centros con menor volumen de pacientes, puesto que los resultados, en el inicio de la técnica, son similares en ambos tipos de centros.

\section{REFERENCIAS}

1. Clayman RV, Kavousi LR, Soper NJ, et Al. Laparoscopic nephrectomy: inicial case report. J. Urol. 1991. 146:278-282.

2. Rassweiler J, Sentker L, Seemann O, Hatzinger M, Rumpelt HJ. Laparoscopic radical prostatectomy with the Heibron technique: an analysis of the first 180 cases. J Urol. 2001;166(6): 2101-2108.

3. Gregori A, Simonato A, Lisian A, Bozzola A, Galli S, Gaboardi F. Laparoscopic radical prostatectomy: peroperative complications in an inicial and consecutive series of 80 cases. Eur Urol. 2003;44:190-194.

4. Stolzenburg J, Do M, Rabenalt R, Pfeifer H, Horn L, Truss MC, Jonas U, Drschner W. Endoscopic extraperitoneal radical prostatectomy: initial experience after 70 procedures. J Urol. 2003; 6:2066-2071.

5. Cecchini LL, Areal J, Saladié JM. Prostatectomía radical laparoscópica. Revisión del primer año. Arch Esp Urol. 2003;56(3): 287-293.

6. Peña JA, Gonzalez JL, Prera A, Abad C, Graells A, Descalzo MC. Prostatectomía radical laparoscópica extraperitoneal. Primeros 50 casos en un centro comarcal. Análisis de la curva de aprendizaje. Actas Urol Esp. 2007;31(7):732-737.

7. Cansino JR, Martínes-Pñeiro L, Alonso S, Pacios JC, Núñez C, Cisneros J, Dela Peña JJ. Estado actual de la prostatectomía radical laparoscópica. Revisión de la literatura. Nuestra experiencia. Endourología. Silmi A, Moreno J. Endourología, laparoscopia y terapia mínimamente invasiva en urología. Clínicas urológicas de la complutense. Madrid. 2005;11:561-581

8. Guillonneau B, El Fettouh H, Baumert H, Cathelineau X, Doublet JD, Fromont G, et al. Laparoscopic radical prostatectomy: oncologicla evaluation after 1000 cases at the Montsouris Institute. J Urol. 2003;169:1261-1266.

9. Rhee HK, Triaca V, Sorcini A, Tuerk IA. Transpreitoenal laparoscopica radical prostatectomy: descending technique. J Endourol. 2004; 18:601-604

10. Rassweiler J, Marrero R, Hammady A, Erdogru T, Teber D, Frede T. Transperitoneal laparoscopic radical prostatectomy: ascending technique. J Endourol. 2004;18:593-600. 
11. Guillonneau B, Cathelineau X, Doublet J, Baumert H, Valencien G. Laparoscopic radical prostatecomy: assesment alter 550 procedures. Crit Rev Oncol Hematol. 2002;43:123-133.

12. Fromont G, Baumert H, Cathelineau X, Rozet F, Validire P, Vallancien G. Intraoperative frozn section análisis during nerve sparing laparoscopic radical prostatectomy: feasibility study. J Urol. 2003;170:1843-1846.

13. Ruiz L, Salomón L, Hoznek A, Vordos D, Yiou R, De la Taille A, et al. Comparison of early oncologic results of laparoscopic radical prostatectomy by extraperitoneal versus tranperitoneal approach. Eur Urol. 2004; 46:50-56.

14. Salomon L, Anastasiadis AG, Katz R, De la Taille A, Saint F, Vordos D, et al. Urinary continence and erectile function: a prospective evaluation of functional results after radical laparoscopic prostatectomy. Eur Urol. 2002;42:338-343.

15. Anastasiadis AG, Salomon L, Katz R, Hoznek A, Chopin D, Abbou CC. Radical retropubic versus laparoscopic prostatectomy: a comparison of functional outcome. Urology. 2003;62: 292-297.

16. Rassweiler J, Schulze M, Teber D, Seemann O, Frede T. Laparoscopic raical prostatectomy: functional and oncological outcomes. Curr Opin Urol. 2004;14:75-82.

17. Rozet F, Galiano M, Catelineau X, Barret E, Cathala N, Vallancien G. Extraperitoneal laparoscopic radical prostatectomy: a prospective evaluation of 600 cases. J Urol. 2005;174: 908911.

18. Roumeguere T, Bollens R, Bossche Mv, Rochet D, Bialek D, Hoffman P, et al. Radical prostatectomy: a prospective comparison of oncological and functional results between open and laparoscopic approaches. World J Urol. 2003;20:360-366.
19. Stolzenburg JU, Rrabenalt, Do M, Ho K, Dorscner W, Waldkirch E, et al. Endoscopic extraperitoneal radical prostatectomy: oncological and functional results alter 700 procedures. J Urol. 2005; 17:785-790.

20. Menon M, Shrivastava FA, Sarle R, Hemal A, Tewari A. Vattikti Intitute prostatectomy: a single-team experience of 100 cases. J Endourol. 2003;17:785-790.

21. Curto F, Benijts J, Pansadoro A, Barmoshe S, Hoepffner JL, Mugnier C, Piechaud T, Gaston R. Nerve sparing laparoscopic radical prostatectomy. Eur Urol. 2006;49:344-352.

22. Raventós CX, Gómez E, Cecchini L, Trilla E, Orsola A, Planas J, De Torre I, Morote J. Prostatectomía radical laparoscópica versus abierta. Actas Urol Esp. 2007;31(2):141-145.

23. Miller J, Smith A, Kouba E, Wallen E, Pruthi R. Prospective evaluation of short-term impact and recovery of health related quality of life in men undergoing robotic assisted laparoscopic radical prostatectomy versus open radical prostatectomy. J Urol. 2007;178(3):854-859.

24. Rassweiler J, Schulze M, Teber D, Seemann O, Frede T. Laparoscopic radical prostatectomy: functional and oncological outcomes. Curr Opin Urol. 2004;14(2):75-82.

Correpondencia autor: Dr. M. Crego Tapias

Servicio de Urología. Hospital de Granollers

Avda. Francesc Ribas, s/n - 08400 Granollers (Barcelona)

Tel.: 938425000

E-mail autor: marccrego@gmail.com

Información artículo: Original - Cáncer de próstata

Trabajo recibido: marzo 2008

Trabajo aceptado: abril 2008 